\title{
The association between renal tumour scoring system components and complications of partial nephrectomy
}

\author{
Darren Desantis, MD, FRCSC; ; Luke T. Lavallée, MD, FRCSC; ${ }^{*}$ Kelsey Witiuk, PhD; ${ }^{\dagger}$ Ranjeeta Mallick, PhD; ${ }^{\dagger}$ \\ Fadi Kamal, MD, FRCSC; ${ }^{*}$ Dean Fergusson, PhD; ${ }^{\dagger}$ Christopher Morash, MD, FRCSC; ${ }^{*}$ \\ Ilias Cagiannos, MD, FRCSC,* Rodney H. Breau, MSC, MD, FRCSC*
}

*Department of Surgery, Division of Urology, Ottawa, ON; †Clinical Epidemiology Program, Ottawa Hospital Research Institute, Ottawa, ON

See related article on page 46.

Cite as: Can Urol Assoc J 2015;9(1-2):39-45. htrp://dx.doi.org/10.5489/cuai.2303 Published online February 5, 2015.

\section{Abstract}

Introduction: We evaluate the associations between 3 renal tumour scoring systems and their components with perioperative complications of partial nephrectomy.

Methods: A consecutive cohort of partial nephrectomy patients was analyzed. Patient characteristics were abstracted from medical records. PADUA scores (preoperative aspects and dimensions used for anatomic classification), RENAL (radius exophyic/endophytic nearness anterior/posterior location scoring) nephrometry scores, and Centrality index (C-index) were determined from preoperative axial images by 2 independent reviewers. Cases were evaluated for postoperative complications up to 30 days after surgery. Pre-specified complication definitions were used for 33 potential medical and surgical complications. Unadjusted and adjusted associations between overall scores, individual components, and complications were determined using log binomial regression.

Results: In total, 118 patients were included in the study. Of these, 36 (30.5\%) surgical complications occurred in 27 (22.9\%) patients. Fourteen $(11.9 \%)$ were Clavien grade $\geq 3$. Overall PADUA score was significantly associated with surgical and overall complications after adjusting for potential confounders. Among all components of the 3 scoring systems, only tumour diameter and exophytic/ endophytic nature of the tumour were significantly associated with complications after adjusting for the other components of the respective scoring system $(p<0.05)$.

Conclusions: Renal tumour scoring systems may help predict the risk of complications after partial nephrectomy. Further refinement of current systems is required. A first step would be to include only components that are significantly associated with complications.

\section{Introduction}

Partial nephrectomy for small renal tumours is preferred to radical nephrectomy because the procedure preserves renal function with comparable oncologic outcomes. ${ }^{1-4}$ However, partial nephrectomy is associated with a higher risk of perioperative complications compared to radical nephrectomy; therefore, choosing which procedure to perform should be based on balancing risks and benefits for individual patients. ${ }^{5,6}$

Three different scoring systems have been proposed to objectively characterize renal tumours from cross-sectional imaging: RENAL Nephrometry Score (RENAL), PADUA Score (PADUA), and Centrality index (C-index). ${ }^{7-9}$ The RENAL and PADUA systems assign a score to individual tumour characteristics, which are summed to determine an overall score and risk group. The C-index method uses tumour size and proximity to the centre of the kidney to calculate a number that represents tumour complexity. Thus far, renal tumour scoring systems have been related to treatment choices, ischemia time, postoperative renal function, histologic aggressiveness, and complications. ${ }^{10-18}$ To the best of our knowledge no study has successfully related the 3 scoring systems and their individual components to postoperative complications.

Despite potential benefits, many surgeons may not use these systems in clinical practice because calculating tumour scores take time and the advantage of these systems over clinical judgment is unknown. Indeed, some tumour factors may be more important to predict surgical risk and a more parsimonious scoring model may have similar performance. ${ }^{18}$ The objective of this study was to apply the scoring systems to a cohort of patients receiving partial nephrectomy and to determine associations between overall scores and individual component scores with postoperative complications. 
Desantis et al.

\section{Methods}

Institutional ethics board approval was obtained to review patients who received partial nephrectomy at The Ottawa Hospital. We recorded patient characteristics, including age, gender, preoperative and postoperative renal function, diabetes, hypertension, smoking history, and heart disease. The most recent preoperative axial imaging (computed tomography $[\mathrm{CT}]$ or magnetic resonance imaging [MRI]) was reviewed by 2 independent physicians (DD, LL) and tumour characteristics were recorded as defined by each of the 3 tumour scoring systems (RENAL, PADUA, C-index).

\section{Tumour characteristics}

Each component of the 3 scoring systems was recorded individually. These tumour features were then used to calculate overall scores. RENAL score includes: tumour size (R), exophytic/endophytic $(E)$, nearness to collecting system/renal sinus $(\mathrm{N})$, anterior/posterior location $(\mathrm{A})$, and location relative to polar lines (L). ${ }^{7}$ PADUA score includes: tumour diameter, location relative to polar lines, anterior/posterior location, medial/lateral location, collecting system association, renal sinus association, and exophytic/endophytic extent. ${ }^{8}$ RENAL and PADUA scores include some of the same tumour components, however they differ in their definitions of some components (e.g., location relative to polar lines). C-index is a calculation based on measurements from axial imaging, including tumour size and position relative to the centre of the kidney (centrality). ${ }^{9}$ More detailed descriptions of the scoring systems can be found in the original articles. ${ }^{7-9}$

\section{Study outcomes}

Complications within 30 days of surgery were abstracted from operative reports, discharge summaries, follow-up visits, postoperative imaging, and laboratory investigations. Complications were divided into surgical and medical (nonsurgical) and classified according to the Clavien system. ${ }^{19}$ Predefined surgical complications included death, surgical re-exploration, conversion to open, bleeding necessitating transfusion, urine leak, dialysis, lymphocele, pyelonephritis, perinephric abscess, retroperitoneal hematoma, wound infection, urinary tract infection, pneumothorax, pseudoaneurysm, and bowel perforation. Medical complications included cardiovascular (new-onset hypertension, angina, myocardial infarction, new onset arrhythmia, congestive heart failure), pulmonary (symptomatic atelectasis, pneumonia, respiratory failure), thromboembolic (deep vein thrombosis, pulmonary embolism, cerebrovascular accident), gastrointestinal (ileus, clostridium difficile infection, pancreatitis), and metabolic (hyperkalemia, rhabdomylosis).

\section{Statistical analysis}

A univariable log binomial regression analysis was performed to determine the association between patient, operative, and tumour characteristics with postoperative surgical, medical, and overall (surgical and medical) complications. Baseline patient characteristics used for analysis included age, gender, diabetes, hypertension, heart disease, and smoking history. Operative characteristics included kidney laterality, surgical approach, ischemia time, and estimated blood loss. Tumour characteristics included RENAL nephrometry score and risk groups, PADUA score and risk groups, and C-index score. In addition, the association between each component of each scoring system and complications was evaluated.

A multivariable analysis was performed to determine the association between each renal tumour scoring system overall score and complications, while adjusting for patient factors that were statistically significantly associated with the outcome of interest in univariable analysis. A second multivariable analysis was performed to determine the association between the individual components of each renal tumour scoring system with surgical complications, while adjusting for the other components of the respective scoring system. Associations were reported as relative risks with 95\% confidence intervals. SAS software version 9.4 for Windows was used for analyses (SAS Institute, Cary, NC).

\section{Results}

In total, 118 patients were included in this analysis (Table 1 ). A total of 36 (30.5\%) surgical complications occurred in 27 $(22.9 \%)$ patients. Fourteen $(11.9 \%)$ were Clavien grade $\geq 3$ (Table 2) ${ }^{19}$ No deaths occurred. The most common surgical complication was bleeding necessitating transfusion, which occurred in $10(8.5 \%)$ patients. There were 49 (41.5\%) medical complications recorded in 37 (31.4\%) patients, of which $4(3.4 \%)$ were Clavien grade $\geq 3$ (Table 2$)$. The most common medical complication was atelectasis, occurring in 14 $(11.7 \%)$ patients.

We recorded associations between patient, surgical, and tumour factors with surgical, non-surgical, and overall complications (Table 3). Factors significantly associated with surgical complications included increased age (RR 1.02, $95 \% \mathrm{Cl} 1.00-1.05, p=0.03)$, increased total ischemia time (RR 1.03, 95\% Cl 1.00-1.05, $p=0.03$ ), increased RENAL score per 1 unit (RR 1.25, 95\% Cl 1.01-1.55, $p=0.04)$, and increased PADUA score per 1 unit (RR 1.37, 95\% Cl 1.11-1.69, $p=0.004)$. Overall PADUA score was significantly associated with Clavien $\geq 3$ complications (RR 1.39 $95 \% \mathrm{Cl} 1.05-1.84, p=0.02)$. C-index was not significantly associated with surgical $(p=0.32)$, non-surgical $(p=0.74)$ or overall ( $p=0.82)$ complications. A statistically significant difference was also noted in high versus low com- 


\begin{tabular}{|c|c|}
\hline Demographics & $\mathbf{N}$ (SD or \%) \\
\hline \multicolumn{2}{|l|}{ Patient characteristics } \\
\hline Age (years) & $61.4(12.57)$ \\
\hline \multicolumn{2}{|l|}{ Sex } \\
\hline Male & $60(50.8 \%)$ \\
\hline Female & $58(49.2 \%)$ \\
\hline Diabetes & $18(15.3 \%)$ \\
\hline Hypertension & $64(52.4 \%)$ \\
\hline Heart disease & $38(32.2 \%)$ \\
\hline Smoking history & $66(55.9 \%)$ \\
\hline \multicolumn{2}{|l|}{ Operative characteristics } \\
\hline \multicolumn{2}{|l|}{ Kidney } \\
\hline Right & $54(45.8 \%)$ \\
\hline Left & $64(54.2 \%)$ \\
\hline \multicolumn{2}{|l|}{ Surgical approach } \\
\hline Open & $97(82.2 \%)$ \\
\hline Laparoscopic & $21(17.8 \%)$ \\
\hline Estimated blood loss (cc) & $326(553)$ \\
\hline \multicolumn{2}{|l|}{ Tumour characteristics } \\
\hline \multicolumn{2}{|l|}{ Tumour location } \\
\hline Anterior & $48(40.7 \%)$ \\
\hline Posterior & $44(37.3 \%)$ \\
\hline Indeterminate & $26(22 \%)$ \\
\hline Tumour diameter (cm) & $2.64(1.38)$ \\
\hline Positive margin & $4(3.4 \%)$ \\
\hline \multicolumn{2}{|l|}{ Tumour pathology } \\
\hline Malignant & $90(76.3 \%)$ \\
\hline Benign & $28(23.7 \%)$ \\
\hline RENAL nephrometry score (mean) & $6.7(1.58)$ \\
\hline PADUA score (mean) & $8.5(1.52)$ \\
\hline C-index (mean) & $3.8(2.00)$ \\
\hline
\end{tabular}

AS: active surveillance; PSA: prostate-specific antigen; PSAD: PSA density;

IQR: interquartile range; 5ARI: 5-alpha reductase inhibitor; DRE: digital rectal exam; TRUS: transrectal ultrasound.

plexity RENAL risk groups (RR 3.63, 95\% Cl 1.34-9.81, $p=0.01$ ) and high versus moderate risk groups (RR 2.75, $95 \% \mathrm{Cl} 1.11-6.81, p=0.004)$. Similar associations were observed for the PADUA risk groups; however, these were not statistically significant. The presence of preoperative diabetes (RR 1.79, 95\% Cl 1.02-3.12, $p=0.04$ ) and longer total ischemia time (RR 1.02, 95\% Cl 1.00-1.04, $\mathrm{p}=0.01$ ) were associated with non-surgical complications. RENAL, PADUA, and C-index scores were not significantly associated with medical complications. Patient age (RR 1.02, $95 \% \mathrm{Cl} 1.00-1.03, p=0.02)$, longer total ischemia time (RR 1.01, 95\% Cl 1.01-1.02, $p=0.002$ ), RENAL score (RR 1.11,95\% Cl 1.00-1.24, $p=0.05$ ), and PADUA score (RR $1.16,95 \% \mathrm{Cl} 1.04-1.30, p=0.01$ ) were significantly associated with overall complications.

In univariable analysis, individual score components (tumours $>4 \mathrm{~cm}$, endophytic tumours, and tumours near the collecting system and renal sinus) were significantly associ-
Table 2. Description of surgical and medical complications in a cohort of 118 patients receiving partial nephrectomy. Clavien $\geq 3$ complications are indicated. ${ }^{19}$

\begin{tabular}{|c|c|c|}
\hline Complication & $\begin{array}{c}\text { Any Clavien } \\
\text { grade, N (\%) }\end{array}$ & $\begin{array}{c}\text { Clavien } \\
\text { grade } \geq 3, \mathbf{N} \\
(\%)\end{array}$ \\
\hline Surgical complications & $36(30.5 \%)$ & $14(11.9 \%)$ \\
\hline Transfusion & $10(8.5 \%)$ & - \\
\hline Hemodialysis & $5(4.2 \%)$ & $5(4.2 \%)$ \\
\hline Retroperitoneal hematoma & $5(4.2 \%)$ & $2(4.2 \%)$ \\
\hline Urine leak & $4(3.4 \%)$ & $3(2.5 \%)$ \\
\hline Urinary tract infection & $4(3.4 \%)$ & - \\
\hline Wound infection & $2(1.7 \%)$ & - \\
\hline Pseudoaneurysm & $2(1.7 \%)$ & $2(1.7 \%)$ \\
\hline Pyelonephritis & $1(0.8 \%)$ & - \\
\hline Perinephric abcess & $1(0.8 \%)$ & $1(0.8 \%)$ \\
\hline Pneumothorax & $1(0.8 \%)$ & - \\
\hline Bowel perforation & $1(0.8 \%)$ & $1(0.8 \%)$ \\
\hline Re-exploration & - & - \\
\hline Conversion to open procedure & - & - \\
\hline Lymphocele & - & - \\
\hline Death & - & - \\
\hline Non-surgical complications & $49(41.5 \%)$ & $4(3.4 \%)$ \\
\hline Atelectasis & $14(11.7 \%)$ & - \\
\hline Hypertension & $13(11.0 \%)$ & - \\
\hline lleus & $5(4.2 \%)$ & - \\
\hline Hyperkalemia & $5(4.2 \%)$ & - \\
\hline Arrhythmia & $3(2.5 \%)$ & - \\
\hline Congestive heart failure & $3(2.5 \%)$ & $3(2.5 \%)$ \\
\hline Pneumonia & $3(2.5 \%)$ & - \\
\hline Angina & $1(0.8 \%)$ & - \\
\hline Myocardial infarction & $1(0.8 \%)$ & $1(0.8 \%)$ \\
\hline Clostridium difficile infection & $1(0.8 \%)$ & - \\
\hline Pulmonary embolism & - & - \\
\hline Respiratory failure & - & - \\
\hline Deep vein thrombosis & - & - \\
\hline Pancreatitis & - & - \\
\hline Rhabdomylosis & - & - \\
\hline
\end{tabular}

ated with increased surgical complications (Table 4). After adjusting for the other components in the respective scoring systems (Table 4, Fig. 1, Fig. 2), tumour size $>4 \mathrm{~cm}$ and endophytic tumours remained significantly associated with surgical complications. The location of the tumour relative to the polar lines (by both RENAL and PADUA definitions) showed an inverse association with surgical complications.

The association between overall scoring system score and complications was adjusted for patient factors that were statistically significant in univariable analysis: age (surgical complications), diabetes (medical complications), age and diabetes (overall complications). After adjustment, overall PADUA score was significantly associated with surgical and overall complications (Table 5). 
Desantis et al.

\begin{tabular}{|c|c|c|c|c|c|c|c|c|}
\hline \multirow[b]{3}{*}{ Variable } & \multicolumn{8}{|c|}{ Postoperative complications by 30 days } \\
\hline & \multicolumn{2}{|c|}{ Surgical } & \multicolumn{2}{|c|}{$\begin{array}{c}\text { Medical } \\
\text { (non-surgical) }\end{array}$} & \multicolumn{2}{|c|}{ Overall } & \multicolumn{2}{|c|}{ Clavien $\geq 3$} \\
\hline & $\begin{array}{c}\text { RR } \\
(95 \% \mathrm{Cl})\end{array}$ & $p$ value & $\begin{array}{c}\mathbf{R R} \\
(95 \% \mathrm{Cl})\end{array}$ & $p$ value & $\begin{array}{c}\mathbf{R R} \\
(95 \% \mathrm{Cl})\end{array}$ & $p$ value & $\begin{array}{c}\mathbf{R R} \\
(95 \% \mathrm{Cl})\end{array}$ & $p$ value \\
\hline Age (years) & $\begin{array}{c}1.02 \\
(1.00-1.05)\end{array}$ & 0.03 & $\begin{array}{c}1.01 \\
(0.99-1.04)\end{array}$ & 0.23 & $\begin{array}{c}1.02 \\
(1.00-1.03)\end{array}$ & 0.02 & - & - \\
\hline Gender (F vs. M) & $\begin{array}{c}0.58 \\
(0.25-1.32)\end{array}$ & 0.19 & $\begin{array}{c}0.97 \\
(0.55-1.72)\end{array}$ & 0.92 & $\begin{array}{c}0.77 \\
(0.50-1.19)\end{array}$ & 0.24 & - & - \\
\hline Diabetes & $\begin{array}{c}0.69 \\
(0.23-2.07)\end{array}$ & 0.51 & $\begin{array}{c}1.79 \\
(1.02-3.12)\end{array}$ & 0.04 & $\begin{array}{c}1.45 \\
(0.98-2.14) \\
\end{array}$ & 0.06 & - & - \\
\hline Hypertension & $\begin{array}{c}1.23 \\
(0.62-2.41) \\
\end{array}$ & 0.55 & $\begin{array}{c}1.56 \\
(0.88-2.75) \\
\end{array}$ & 0.13 & $\begin{array}{c}1.38 \\
(0.94-2.03) \\
\end{array}$ & 0.10 & - & - \\
\hline Heart disease & $\begin{array}{c}1.45 \\
(0.75-2.81) \\
\end{array}$ & 0.27 & $\begin{array}{c}1.14 \\
(0.66-1.98) \\
\end{array}$ & 0.64 & $\begin{array}{c}1.29 \\
(0.89-1.85) \\
\end{array}$ & 0.17 & - & - \\
\hline Smoking & $\begin{array}{c}1.15 \\
(0.58-2.25)\end{array}$ & 0.69 & $\begin{array}{c}1.29 \\
(0.74-2.26)\end{array}$ & 0.36 & $\begin{array}{c}1.29 \\
(0.88-1.90)\end{array}$ & 0.20 & - & - \\
\hline Kidney (L vs. R) & $\begin{array}{c}0.78 \\
(0.40-1.52) \\
\end{array}$ & 0.47 & $\begin{array}{c}1.76 \\
(0.98-3.16) \\
\end{array}$ & 0.06 & $\begin{array}{c}1.20 \\
(0.82-1.74) \\
\end{array}$ & 0.35 & - & - \\
\hline Approach (Lap vs. Open) & $\begin{array}{c}0.80 \\
(0.31-2.08)\end{array}$ & 0.65 & $\begin{array}{c}0.26 \\
(0.07-1.01)\end{array}$ & 0.05 & $\begin{array}{c}0.53 \\
(0.26-1.07)\end{array}$ & 0.08 & - & - \\
\hline Ischemia time (per minute) & $\begin{array}{c}1.03 \\
(1.00-1.05) \\
\end{array}$ & 0.03 & $\begin{array}{c}1.02 \\
(1.00-1.04) \\
\end{array}$ & 0.01 & $\begin{array}{c}1.01 \\
(1.01-1.02) \\
\end{array}$ & 0.002 & - & - \\
\hline C-index decrease & $\begin{array}{c}1.11 \\
(0.90-1.42)\end{array}$ & 0.32 & $\begin{array}{c}1.02 \\
(0.89-1.17)\end{array}$ & 0.74 & $\begin{array}{c}1.01 \\
(0.91-1.12)\end{array}$ & 0.82 & $\begin{array}{c}1.04 \\
(0.81-1.35)\end{array}$ & 0.76 \\
\hline $\begin{array}{l}\text { RENAL nephrometry score } \\
\text { (unit) }\end{array}$ & $\begin{array}{c}1.25 \\
(1.01-1.55) \\
\end{array}$ & 0.04 & $\begin{array}{c}1.07 \\
(0.91-1.25) \\
\end{array}$ & 0.41 & $\begin{array}{c}1.11 \\
(1.00-1.24) \\
\end{array}$ & 0.05 & $\begin{array}{c}1.23 \\
(0.92-1.64) \\
\end{array}$ & 0.17 \\
\hline \multicolumn{9}{|c|}{ RENAL nephrometry risk groups } \\
\hline Moderate vs. low & $\begin{array}{c}1.32 \\
(0.64-2.73) \\
\end{array}$ & 0.46 & - & - & $\begin{array}{c}1.34 \\
(0.89-2.00) \\
\end{array}$ & 0.16 & - & - \\
\hline High vs. low & $\begin{array}{c}3.63 \\
(1.34-9.81) \\
\end{array}$ & 0.01 & - & - & $\begin{array}{c}1.63 \\
(0.69-3.89)\end{array}$ & 0.27 & - & - \\
\hline High vs. moderate & $\begin{array}{c}2.75 \\
(1.11-6.81) \\
\end{array}$ & 0.03 & - & - & $\begin{array}{c}1.22 \\
(0.53-2.80) \\
\end{array}$ & 0.64 & - & - \\
\hline $\begin{array}{l}\text { PADUA score } \\
\text { (unit) }\end{array}$ & $\begin{array}{c}1.37 \\
(1.11-1.69)\end{array}$ & 0.004 & $\begin{array}{c}1.06 \\
(0.90-1.24)\end{array}$ & 0.50 & $\begin{array}{c}1.16 \\
(1.04-1.30)\end{array}$ & 0.01 & $\begin{array}{c}1.39 \\
(1.05-1.84)\end{array}$ & 0.02 \\
\hline \multicolumn{9}{|c|}{ PADUA Risk Groups } \\
\hline Moderate vs. low & $\begin{array}{c}1.21 \\
(0.45-3.22)\end{array}$ & 0.71 & - & - & $\begin{array}{c}1.31 \\
(0.77-2.21) \\
\end{array}$ & 0.32 & - & - \\
\hline High vs. low & $\begin{array}{c}2.33 \\
(0.92-5.86) \\
\end{array}$ & 0.07 & - & - & $\begin{array}{c}1.62 \\
(0.96-2.73) \\
\end{array}$ & 0.07 & - & - \\
\hline High vs. moderate & $\begin{array}{c}1.93 \\
(0.94-3.95) \\
\end{array}$ & 0.07 & - & - & $\begin{array}{c}1.24 \\
(0.84-1.82) \\
\end{array}$ & 0.29 & - & - \\
\hline
\end{tabular}

\section{Discussion}

Accurately characterizing renal tumours may improve preoperative patient counselling and may assist surgeons in selecting the best surgical or ablative approach. This study has several noteworthy findings. PADUA score was independently associated with surgical complications. However, some components, such as tumour size and tumour depth within the kidney, seem more predictive of complications than others. Furthermore, polar versus mid-kidney location for both the PADUA and RENAL systems had the inverse relationship to perioperative complications.

Overall, these data indicate that the proposed scoring systems are able to objectively characterize tumours and stratify them for risk of surgical complications. Other studies have examined the associations between scoring systems 


\begin{tabular}{|c|c|c|c|c|c|c|c|c|}
\hline \multirow[b]{3}{*}{ Variable } & \multicolumn{8}{|c|}{ Postoperative complications by 30 days } \\
\hline & \multicolumn{2}{|c|}{ Surgical } & \multicolumn{2}{|c|}{$\begin{array}{c}\text { Medical } \\
\text { (non-surgical) }\end{array}$} & \multicolumn{2}{|c|}{$\begin{array}{c}\text { Overall } \\
\text { (surgincal + medical) }\end{array}$} & \multicolumn{2}{|c|}{ Surgical* } \\
\hline & $\begin{array}{c}\mathrm{RR} \\
(95 \% \mathrm{Cl}) \\
\end{array}$ & $p$ value & $\begin{array}{c}\mathrm{RR} \\
(95 \% \mathrm{Cl}) \\
\end{array}$ & $p$ value & $\begin{array}{c}\mathrm{RR} \\
(95 \% \mathrm{Cl}) \\
\end{array}$ & $p$ value & $\begin{array}{c}\mathrm{RR} \\
(95 \% \mathrm{Cl}) \\
\end{array}$ & $p$ value \\
\hline \multicolumn{9}{|c|}{ RENAL NEPHROMETRY SCORE COMPONENTS } \\
\hline \multicolumn{9}{|l|}{ R-Radius of tumour } \\
\hline$>4$ to $<7 \mathrm{~cm}$ vs. $\leq 4 \mathrm{~cm}$ & $\begin{array}{c}1.82 \\
(0.87-3.81) \\
\end{array}$ & 0.11 & $\begin{array}{c}0.56 \\
(0.20-1.62) \\
\end{array}$ & 0.29 & $\begin{array}{c}1.02 \\
(0.60-1.73) \\
\end{array}$ & 0.94 & $\begin{array}{c}2.53 \\
(1.04-6.10) \\
\end{array}$ & 0.04 \\
\hline \multicolumn{9}{|l|}{ E - Exophytic/Endophytic } \\
\hline$<50 \%$ vs. $\geq 50 \%$ exophytic & $\begin{array}{c}1.99 \\
(0.86-4.60)\end{array}$ & 0.11 & $\begin{array}{c}1.06 \\
(0.60-1.89)\end{array}$ & 0.83 & $\begin{array}{c}1.41 \\
(0.91-2.16)\end{array}$ & 0.12 & $\begin{array}{c}2.33 \\
(1.00-5.47)\end{array}$ & 0.05 \\
\hline $\begin{array}{l}\text { Entirely endophytic vs. }<50 \% \\
\text { exophytic }\end{array}$ & $\begin{array}{c}1.84 \\
(0.64-5.28) \\
\end{array}$ & 0.26 & $\begin{array}{c}1.59 \\
(0.56-4.50) \\
\end{array}$ & 0.38 & $\begin{array}{c}1.38 \\
(0.75-2.53) \\
\end{array}$ & 0.30 & $\begin{array}{c}2.19 \\
(0.65-7.32) \\
\end{array}$ & 0.20 \\
\hline $\begin{array}{l}\text { Entirely endophytic vs. } \geq 50 \% \\
\text { exophytic }\end{array}$ & $\begin{array}{c}3.67 \\
(1.07-12.55) \\
\end{array}$ & 0.04 & $\begin{array}{c}1.69 \\
(0.57-4.99) \\
\end{array}$ & 0.34 & $\begin{array}{c}1.94 \\
(0.99-3.82) \\
\end{array}$ & 0.05 & $\begin{array}{c}5.11 \\
(1.21-21.64) \\
\end{array}$ & 0.03 \\
\hline \multicolumn{9}{|l|}{ N - Nearness (mm) } \\
\hline $5-6$ vs. $\geq 7$ & $\begin{array}{c}2.28 \\
(0.84-6.19) \\
\end{array}$ & 0.11 & $\begin{array}{c}1.14 \\
(0.49-2.67) \\
\end{array}$ & 0.77 & $\begin{array}{c}1.43 \\
(0.83-2.49) \\
\end{array}$ & 0.20 & $\begin{array}{c}2.05 \\
(0.77-5.49) \\
\end{array}$ & 0.15 \\
\hline$\leq 4$ vs. $\geq 7$ & $\begin{array}{c}2.14 \\
(0.95-4.81) \\
\end{array}$ & 0.06 & $\begin{array}{c}1.29 \\
(0.72-2.30) \\
\end{array}$ & 0.40 & $\begin{array}{c}1.45 \\
(0.96-2.20) \\
\end{array}$ & 0.08 & $\begin{array}{c}1.20 \\
(0.46-3.09) \\
\end{array}$ & 0.71 \\
\hline$\leq 4$ vs. $5-6$ & $\begin{array}{c}0.94 \\
(0.41-2.18)\end{array}$ & 0.89 & $\begin{array}{c}1.13 \\
(0.50-2.55) \\
\end{array}$ & 0.77 & $\begin{array}{c}1.01 \\
(0.62-1.66)\end{array}$ & 0.97 & $\begin{array}{c}0.58 \\
(0.23-1.51) \\
\end{array}$ & 0.27 \\
\hline A - Posterior vs. Anterior & $\begin{array}{c}1.14 \\
(0.92-1.41) \\
\end{array}$ & 0.22 & $\begin{array}{c}0.94 \\
(0.75-1.18) \\
\end{array}$ & 0.59 & $\begin{array}{c}1.00 \\
(0.87-1.16) \\
\end{array}$ & 0.99 & - & - \\
\hline \multicolumn{9}{|l|}{ L - Location relative to polar lines } \\
\hline Crosses vs. above & $\begin{array}{c}1.25 \\
(0.59-2.67)\end{array}$ & 0.56 & $\begin{array}{c}2.00 \\
(1.04-3.86)\end{array}$ & 0.04 & $\begin{array}{c}1.60 \\
(1.03-2.47)\end{array}$ & 0.03 & $\begin{array}{c}1.03 \\
(0.50-2.13)\end{array}$ & 0.94 \\
\hline Between vs. above & $\begin{array}{c}0.85 \\
(0.36-2.02) \\
\end{array}$ & 0.71 & $\begin{array}{c}1.34 \\
(0.64-2.80) \\
\end{array}$ & 0.44 & $\begin{array}{c}1.15 \\
(0.70-1.89) \\
\end{array}$ & 0.59 & $\begin{array}{c}0.82 \\
(0.35-1.92) \\
\end{array}$ & 0.65 \\
\hline Between vs. crosses & $\begin{array}{c}0.68 \\
(0.29-1.59) \\
\end{array}$ & 0.38 & $\begin{array}{c}0.67 \\
(0.36-1.24) \\
\end{array}$ & 0.20 & $\begin{array}{c}0.72 \\
(0.47-1.10) \\
\end{array}$ & 0.13 & $\begin{array}{c}0.80 \\
(0.35-1.85) \\
\end{array}$ & 0.60 \\
\hline \multicolumn{9}{|c|}{ PADUA SCORE COMPONENTS } \\
\hline $\begin{array}{l}\text { Tumour diameter } \\
>4 \text { to }<7 \mathrm{~cm} \text { vs. } \leq 4 \mathrm{~cm}\end{array}$ & $\begin{array}{c}1.82 \\
(0.87-3.81) \\
\end{array}$ & 0.11 & $\begin{array}{c}0.56 \\
(0.20-1.62) \\
\end{array}$ & 0.29 & $\begin{array}{c}1.02 \\
(0.60-1.73) \\
\end{array}$ & 0.94 & $\begin{array}{c}2.53 \\
(1.04-6.10) \\
\end{array}$ & 0.04 \\
\hline $\begin{array}{l}\text { Polar location } \\
\text { Between vs. Above/Below polar line }\end{array}$ & $\begin{array}{c}0.90 \\
(0.46-1.76)\end{array}$ & 0.75 & $\begin{array}{c}1.66 \\
(0.89-3.10) \\
\end{array}$ & 0.11 & $\begin{array}{c}1.37 \\
(0.91-2.07)\end{array}$ & 0.14 & $\begin{array}{c}0.88 \\
(0.45-1.72)\end{array}$ & 0.70 \\
\hline Posterior vs. Anterior & $\begin{array}{c}1.14 \\
(0.92-1.41) \\
\end{array}$ & 0.22 & $\begin{array}{c}0.94 \\
(0.75-1.18) \\
\end{array}$ & 0.59 & $\begin{array}{c}1.00 \\
(0.87-1.16) \\
\end{array}$ & 0.99 & - & - \\
\hline Medial vs. lateral location & $\begin{array}{c}1.75 \\
(0.91-3.37) \\
\end{array}$ & 0.10 & $\begin{array}{c}0.99 \\
(0.57-1.71) \\
\end{array}$ & 0.96 & $\begin{array}{c}1.32 \\
(0.92-1.89) \\
\end{array}$ & 0.13 & $\begin{array}{c}1.63 \\
(0.78-3.41) \\
\end{array}$ & 0.20 \\
\hline $\begin{array}{l}\text { Collecting system association } \\
\text { (yes vs. no) }\end{array}$ & $\begin{array}{c}1.98 \\
(1.04-3.77) \\
\end{array}$ & 0.04 & $\begin{array}{c}1.19 \\
(0.68-2.08) \\
\end{array}$ & 0.55 & $\begin{array}{c}1.30 \\
(0.90-1.88) \\
\end{array}$ & 0.16 & $\begin{array}{c}1.63 \\
(0.45-5.92) \\
\end{array}$ & 0.46 \\
\hline Renal sinus association (yes vs. no) & $\begin{array}{c}1.96 \\
(1.01-3.80) \\
\end{array}$ & 0.05 & $\begin{array}{c}1.07 \\
(0.62-1.84) \\
\end{array}$ & 0.81 & $\begin{array}{c}1.27 \\
(0.89-1.83) \\
\end{array}$ & 0.19 & $\begin{array}{c}0.74 \\
(0.20-2.81) \\
\end{array}$ & 0.66 \\
\hline $\begin{array}{l}\text { Exophytic Extent } \\
<50 \% \text { vs. } \geq 50 \% \text { exophytic }\end{array}$ & $\begin{array}{c}1.99 \\
(0.86-4.60) \\
\end{array}$ & 0.11 & $\begin{array}{c}1.06 \\
(0.60-1.89) \\
\end{array}$ & 0.83 & $\begin{array}{c}1.41 \\
(0.91-2.16) \\
\end{array}$ & 0.12 & $\begin{array}{c}2.33 \\
(0.89-6.09) \\
\end{array}$ & 0.09 \\
\hline $\begin{array}{l}\text { Entirely endophytic vs. } \geq 50 \% \\
\text { exophytic }\end{array}$ & $\begin{array}{c}3.67 \\
(1.07- \\
12.55) \\
\end{array}$ & 0.04 & $\begin{array}{c}1.69 \\
(0.57-4.99)\end{array}$ & 0.34 & $\begin{array}{c}1.94 \\
(0.99-3.82)\end{array}$ & 0.05 & $\begin{array}{c}5.94 \\
(1.12-31.43)\end{array}$ & 0.04 \\
\hline $\begin{array}{l}\text { Entirely endophytic vs. }<50 \% \\
\text { exophytic }\end{array}$ & $\begin{array}{c}1.84 \\
(0.64-5.28) \\
\end{array}$ & 0.26 & $\begin{array}{c}1.59 \\
(0.56-4.50)\end{array}$ & 0.38 & $\begin{array}{c}1.38 \\
(0.75-2.53)\end{array}$ & 0.30 & $\begin{array}{c}2.55 \\
(0.61-10.69) \\
\end{array}$ & 0.20 \\
\hline
\end{tabular}




\begin{tabular}{|c|c|c|c|c|c|c|}
\hline \multirow[b]{3}{*}{ Variable } & \multicolumn{6}{|c|}{ Postoperative complications by 30 days } \\
\hline & \multicolumn{2}{|c|}{ Surgical } & \multicolumn{2}{|c|}{ Medical (non-surgical) } & \multicolumn{2}{|c|}{ Overall } \\
\hline & RR (95\% Cl) & $p$ value & RR (95\% Cl) & $p$ value & RR (95\% Cl) & $p$ value \\
\hline RENAL Score & $1.19(0.95-1.50)$ & 0.14 & $1.05(0.90-1.22)$ & 0.57 & $1.10(0.98-1.24)$ & 0.11 \\
\hline PADUA Score & $1.31(1.05-1.65)$ & 0.02 & $1.04(0.88-1.22)$ & 0.66 & $1.12(1.00-1.25)$ & 0.04 \\
\hline C-Index decrease & $1.05(0.85-1.29)$ & 0.64 & $1.00(0.88-1.15)$ & 0.96 & $0.98(0.90-1.08)$ & 0.75 \\
\hline
\end{tabular}

Analyses adjusted for: age (surgical complications), diabetes (medical complications), age and diabetes (overall complications).

and complications during open, laparoscopic, and robotic partial nephrectomy with similar findings. ${ }^{12,18,20-24}$ However, as has been previously suggested, our data suggest current systems can be improved. ${ }^{25}$

One notable difference between this study and most others is the evaluation of individual scoring system components. ${ }^{12,20,22,23}$ Ideally, all elements of a preoperative predictive model for kidney tumours should be associated with important clinical outcomes and should be easy to understand and apply in practice. In this study, larger tumour size and endophytic location were independently associated with surgical complications after adjusting for the other components in the tumour systems. Additional information provided by measuring tumour proximity to the renal sinus and collecting system was small and not statistically significant. A second interesting finding from this study is that tumour location relative to the polar lines was inversely associated with complications. This indicates that this component may detract from the predictive value of PADUA and RENAL systems. If other studies confirm our findings, this component should likely be removed or modified. This may improve the scores' discriminative value, and render them more user-friendly for application in clinical practice.

Our data support the findings of 2 recently published studies which examined different renal tumour scoring meth-

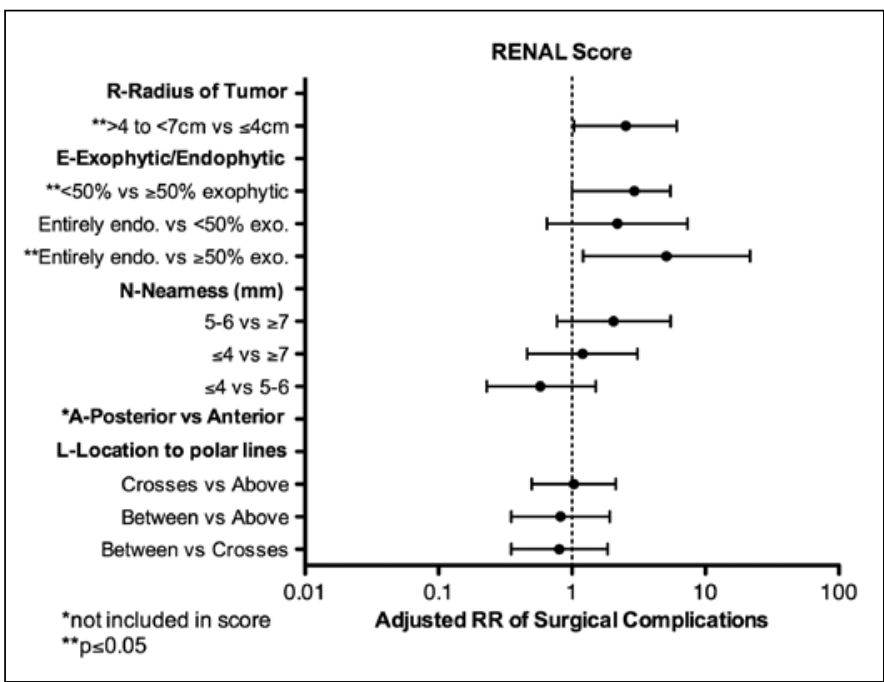

Fig. 1. Associations between components of the RENAL score and adjusted relative risk of surgical complications with $95 \%$ confidence intervals. ods. ${ }^{24,26}$ In the first study, the authors similarly evaluated all 3 scoring systems and found that a novel "RTII score" assessing tumour depth to parenchymal thickness ratio had better discrimination than RENAL or PADUA systems. ${ }^{24}$ In the second study, the authors hypothesized tumour contact with the parenchyma would require more renal parenchymal resection, more transection of large blood vessels, and a more complex reconstruction. They devised a novel measure of tumour complexity incorporating similar components as the RTII score, namely the contact surface area (CSA), and found it associated with partial nephrectomy outcomes including complications. ${ }^{26}$

\section{Conclusion}

Each of these novel scoring methods requires further validation. However considered together with data from our study, these reports suggest a parsimonious and user-friendly score incorporating tumour size and some measure of the tumour's depth/contact with the renal parenchyma can likely perform at least as well as RENAL or PADUA systems.

Competing interests: Dr. Desantis, Dr. Witiuk, Dr. Mallick, Dr, Kamal, Dr. Fergusson, Dr. Lavallée, and Dr. Breau declare no competing financial or personal interests. Dr. Morash is a member of advisory boards for AbbVie, Janssen, Amgen, and Sanofi; he has also received grants from AstraZeneca

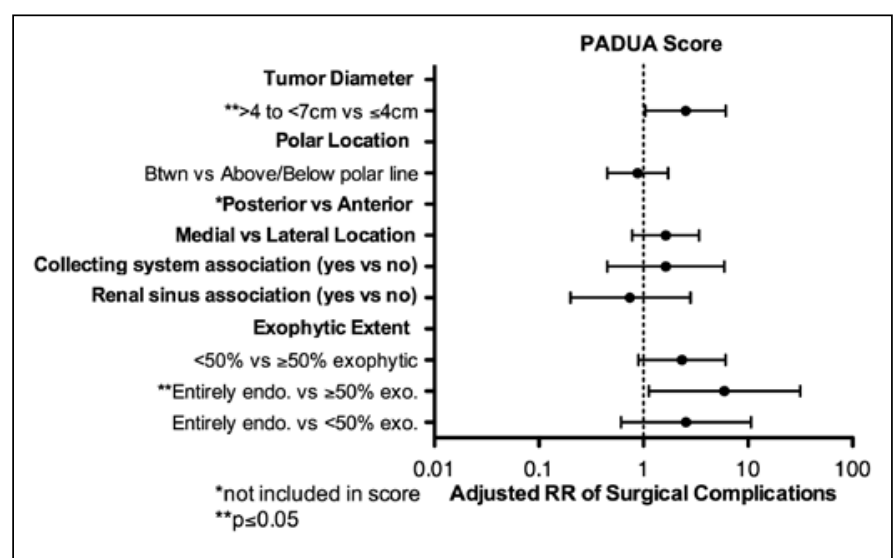

Fig. 2. Associations between PADUA score components and adjusted relative risk of surgical complications with $95 \%$ confidence intervals. 
and Paladin. Dr. Cagiannos is a member of advisory boards for Ferring and Janssen; he has also received grants from Ferring and AbbVie.

This paper has been peer-reviewed.

\section{References}

1. Huang WC, Elkin EB, Levey AS, et al. Partial nephrectomy versus radical nephrectomy in patients with smal renal tumors: Is there a difference in mortality and cardiovascular outcomes? J Urol 2009;181:55-61; discussion 61-2. http://dx.doi.org/10.1016/i.juro.2008.09.017

2. Huang WC, Levey AS, Serio AM, et al. Chronic kidney disease after nephrectomy in patients with renal cortical tumors: A retrospective cohort study. Lancet Oncol 2006;7:735-40. http://dx.doi.org/10.1016/ S1470-2045(06)70803-8

3. Thompson RH, Boorijian SA, Lohse CM, et al. Radical nephrectomy for pTla renal masses may be associated with decreased overall survival compared with partial nephrectomy. I Urol 2008;179:468-71; discussion 472-3. http://dx.doi.org/10.1016/i.juro.2007.09.077

4. Clark ATD, Breau RH, Morash C, et al. Preservation of renal function following partial or radical nephrectomy using 24-hour creatinine clearance. Eur Urol 2008;54:143-9. http://dx.doi.org/10.1016/i. eururo.2008.03.037

5. Lesage $\mathrm{K}$, Joniau $S$, Fransis $\mathrm{K}$, et al. Comparison between open partial and radical nephrectomy for renal tumors: Perioperative outcome and health-related quality of life. Eur Urol 2007;51:614-20. http:// dx.doi.org/10.1016/i.eururo.2006.10.040

6. Van Poppel H, Da Pozzo L, Albrecht W, et al. A prospective, randomised EORTC intergroup phase 3 study comparing the oncologic outcome of elective nephron-sparing surgery and radical nephrectomy for low-stage renal cell carcinoma. Eur Urol 2011;59:543-52. http://dx.doi.org/10.1016/i.eururo.2010.12.013

7. Kutikov A, Uzzo RG. The R.E.N.A.L. nephrometry score: A comprehensive standardized system for quantitating renal tumor size, location and depth. J Urol 2009;182:844-53. http://dx.doi.org/10.1016/i. juro.2009.05.035

8. Ficarra V, Novara $G$, Secco $S$, et al. Preoperative aspects and dimensions used for an anatomical (PADUA) classification of renal tumors in patients who are candidates for nephron-sparing surgery. Eur Urol 2009;56:786-93. http://dx.doi.org/10.1016/i.eururo.2009.07.040

9. Simmons MN, Ching CB, Samplaski MK, et al. Kidney tumor location measurement using the $C$ index method. J Urol 2010;183:1708-13. http://dx.doi.org/10.1016/i.juro.2010.01.005

10. Canter D, Kutikov A, Manley B, et al. Utility of the R.E.N.A.L. nephrometry scoring system in objectifying treatment decision-making of the enhancing renal mass. Urology 2011;78:1089-94. http://dx.doi. org/10.1016/.jurology.2011.04.035

11. Hayn MH, Schwaab T, Underwood W, et al. RENAL nephrometry score predicts surgical outcomes of laparoscopic partial nephrectomy. BJU Int 2011;108:876-81.

12. Okhunov Z, Rais-Bahrami S, George AK, et al. The comparison of three renal tumor scoring systems: C-Index, P.A.D.U.A., and R.E.N.A.L. nephrometry scores. J Endourol 2011;25:1921-4. http://dx.doi. org/10.1089/end.2011.0301
13. Samplaski MK, Hernandez A, Gill IS, et al. C-index is associated with functional outcomes after laparoscopic partial nephrectomy. J Urol 2010;184:2259-63. http://dx.doi.org/10.1016/i.juro.2010.08.031

14. Satasivam P, Sengupta S, Rajarubendra N, et al. Renal lesions with low R.E.N.A.L nephrometry score are associated with more indolent renal cell carcinomas (RCCS) or benign histology: Findings in an Australian cohort. BJU Int 2012;109(Suppl):44-7. http://dx.doi.org/10.1111/j.1464-410X.2012.11046.x

15. Stroup SP, Palazzi K, Kopp RP, et al. RENAL nephrometry score is associated with operative approach for partial nephrectomy and urine leak. Urology 2012;80:151-6. http://dx.doi.org/10.1016/i.urology.2012.04.026

16. Lavallée LT, Desantis D, Kamal F, et al. The association between renal tumor scoring systems and ischemia time during open partial nephrectomy. Can Urol Assoc J 2012;6:1-8.

17. Bruner $B$, Breau $R H$, Lohse $C M$, et al. Renal nephrometry score is associated with urine leak after partial nephrectomy. BJU Int 2011;108:67-72. http://dx.doi.org/10.1111/j.1464-410X.2010.09837.x

18. Hew MN, Baseskioglu B, Barwari K, et al. Critical appraisal of the PADUA classification and assessment of the R.E.N.A.L. nephrometry score in patients undergoing partial nephrectomy. J Urol 2011;186:42-6. http://dx.doi.org/10.1016/i.juro.2011.03.020

19. Dindo D, Demartines N, Clavien P-A. Classification of surgical complications: A new proposal with evaluation in a cohort of 6336 patients and results of a survey. Ann Surg 2004;240:205-13. http://dx.doi. org/10.1097/01.sla.0000133083.54934.ae

20. Tanagho YS, Kaouk JH, Allaf ME, et al. Perioperative complications of robot-assisted partial nephrectomy: Analysis of 886 patients at 5 United States centers. Urology 2013;81:573-9. http://dx.doi. org/10.1016/i.urology.2012.10.067

21. Yeon JS, Son SJ, Lee YJ, et al. The nephrometry score: is it effective for predicting perioperative outcome during robot-assisted partial nephrectomy? Korean J Urol 2014;55:254-9. http://dx.doi.org/10.4111/ kju.2014.55.4.254

22. Long J-A, Arnoux V, Fiard G, et al. External validation of the RENAL nephrometry score in renal tumors treated by partial nephrectomy. BJU Int 2013;111:233-9. http://dx.doi.org/10.1111/j.1464410X.2012.11339.X

23. Mathieu $R$, Verhoest $G$, Droupy $S$, et al. Predictive factors of complications after robot-assisted laparoscopic partial nephrectomy: a retrospective multicentre study. BJU Int 2013;112:E283-9.

24. Nisen $H$, Ruutu $M$, Glücker $E$, et al. Renal tumor invasion index as a novel anatomical classification predicting urological complications after partial nephrectomy. Scand J Urol 2014;48:41-51. http:// dx.doi.org/10.3109/21681805.2013.797491

25. Volpe A, Terrone C. Anatomic classification systems of renal tumors: New, useful tools in renal surgical oncology. Eur Urol 2011;60:731-3. http://dx.doi.org/10.1016/i.eururo.2011.07.038

26. Leslie S, Gill IS, de Castro Abreu AL, et al. Renal tumor contact surface area: A novel parameter for predicting complexity and outcomes of partial nephrectomy. Eur Urol 2014 Mar 19. http://dx.doi. org/10.1016/i.eururo.2014.03.010

Correspondence: Dr. Rodney Breau, Department of Surgery, Division of Urology, Ottawa, ON; rbreau@toh.on.ca 